\title{
Arterial Stiffness Measured as Pulse Wave Velocity is Highly Correlated with Coronary Atherosclerosis in Asymptomatic Patients
}

\author{
Chiu-Shong Liu ${ }^{1,2}$, Chia-Ing Li ${ }^{3}$, Chuen-Ming Shih ${ }^{4}$, Wen-Yuan Lin ${ }^{1,2}$, Chih-Hsueh Lin ${ }^{1,2}$, Shih-Wei Lai ${ }^{1,2}$, \\ Tsai-Chung Li ${ }^{5,6}$, and Cheng-Chieh Lin ${ }^{1,2,6,7}$
}

\author{
${ }^{1}$ Department of Family Medicine, China Medical University Hospital, Taichung, Taiwan \\ ${ }^{2}$ School of Medicine, College of Medicine, China Medical University, Taichung, Taiwan \\ ${ }^{3}$ Department of Medical Research, China Medical University Hospital, Taichung, Taiwan \\ ${ }^{4}$ Preventive Medicine Center, China Medical University Hospital, Taichung, Taiwan \\ ${ }^{5}$ Graduate Institute of Biostatistics, College of Public Health, China Medical University, Taichung, Taiwan \\ ${ }^{6}$ Department of Healthcare Administration, College of Health Science, Asia University, Taichung, Taiwan \\ ${ }^{7}$ School and Graduate Institute of Health Care Administration, College of Public Health, China Medical University, Taichung, Taiwan
}

\begin{abstract}
Aim: Carotid-femoral pulse wave velocity is a well-known predictor of all-cause and cardiovascular mortality. Few studies have evaluated the relationship between brachial-ankle pulse wave velocity (baPWV) and coronary artery disease. We conducted this study to elucidate the relationship between arterial stiffness measured by ba-PWV and coronary atherosclerosis.

Methods: An automatic waveform analyzer was used to measure the ba-PWV. Multidetector computed tomography coronary angiography was used to assess the coronary artery calcium (CAC) score and to detect coronary stenosis. A total of 654 patients, including 358 women and 296 men (mean age, $54.5 \pm 9.4$ years), were recruited during the period March 2005 to June 2008 .

Results: One hundred and twenty-seven patients (19.4\%) had at least one stenotic coronary vessel. Mean ba-PWV and mean CAC scores were significantly higher in the stenotic group than in the normal control $(15.94 \pm 3.07 \mathrm{~m} / \mathrm{s}$ vs. $14.39 \pm 0.98 \mathrm{~m} / \mathrm{s} ; 293.1 \pm 435.9$ vs. $29.8 \pm 110.8$, respectively; both $p<0.001$ ). The adjusted OR for coronary stenosis increased as ba-PWV increased ( $p$ for trend $=$ $0.0001)$. Using ba-PWV $<14.0 \mathrm{~m} / \mathrm{s}$ as the reference group, we found that ba-PWV between 14.0 $18.0 \mathrm{~m} / \mathrm{s}$ and ba-PWV $>18.0 \mathrm{~m} / \mathrm{s}$ were significantly associated with coronary stenosis (OR, 2.48; CI, 1.56-3.93 and OR, 3.16; CI, 1.68-5.95, respectively). The cutoff point at $15.64 \mathrm{~m} / \mathrm{s}$ using the ROC curve showed a sensitivity of $64.5 \%$, specificity of $65.6 \%$, and an AUC of 0.662 in predicting coronary artery stenosis. Ba-PWV had an additional power for correlating coronary artery disease with the Framingham risk score.

Conclusions: Ba-PWV correlated well with coronary atherosclerosis. Lifestyle modification is an efficacious therapeutic intervention for preventing the progression of arterial stiffness. This non-invasive technique can assist in the early detection of cardiovascular disease and should be included in community screening programs.
\end{abstract}

J Atheroscler Thromb, 2011; 18:652-658.

Key words; Atherosclerosis, Coronary artery disease, Pulse wave velocity

Address for correspondence: Cheng-Chieh Lin, Department of Family Medicine, China Medical University Hospital, 2, Yuh-Der Road, Taichung, Taiwan 404

E-mail: cclin@mail.cmuh.org.tw

Received: September 10, 2010

Accepted for publication: January 28, 2011

\section{Introduction}

Although the prevalence of coronary artery disease (CAD) has declined in the United States and in most European countries ${ }^{1)}$, CAD is still the leading cause of death in Taiwan ${ }^{2)}$. Arterial stiffness has been 
shown to be associated with an increased risk of cardiovascular events ${ }^{3,4)}$. Evaluation of arterial stiffness may assist in the early detection of atherosclerosis ${ }^{5)}$. Pulse wave velocity (PWV) is an indicator of arterial stiffness and a marker of vascular damage ${ }^{6-8)}$; however, in most of these studies, central PWV rather than peripheral PWV is normally used as an indicator of arterial stiffness ${ }^{9)}$.

Multidetector row computed tomography (MDCT) is available in many general hospitals and enables accurate noninvasive assessment of coronary artery calcification (CAC) and stenosis ${ }^{10,11)}$. Few studies have evaluated the relationship between peripheral arterial stiffness and CAD. We conducted this study to elucidate the relationship between brachial-ankle pulse wave velocity (ba-PWV) and coronary atherosclerosis using 64-slice MDCT in an asymptomatic general population.

\section{Methods}

\section{Study Subjects}

All of the 763 individuals that underwent a routine health examination for cardiovascular disease at the China Medical University Hospital during the period March 2005 to June 2008 were invited to participate in this study. Brachial-ankle PWV and coronary MDCT were included in the examination. Each participant was asked to complete a structured questionnaire designed to collect basic demographic data, medical history, and lifestyle characteristics.

Participants with a history of myocardial infarction, significant angina pectoris, significant cerebral vascular disease, or documented peripheral arterial disease were excluded from the study. They were all asymptomatic but with one or more risk factors of coronary heart disease. Of the 763 subjects who were invited, 21 patients did not complete the MDCT examination because of arrhythmia such as atrial fibrillation or other causes, 56 had cardiovascular diseases, and 32 did not sign the consent form; therefore, the final study population was composed of 654 individuals (296 men and 358 women; mean age, $54.5 \pm$ 9.4 years).

\section{Measurement of Brachial-Ankle PWV Ankle-Brachial Pressure Index (ABI)}

Brachial-ankle PWV (baPWV) was measured using a volume-plethymographic apparatus (PWV/ ABI; Colin Co., Ltd., Komaki, Japan) in accordance with previously described methodology ${ }^{12,13)}$. This instrument records PWV, blood pressure in the four limbs, electrocardiogram, and heart sounds simultane- ously. In all patients, baPWV was obtained after at least a 5-min rest in a supine position. The interobserver and intraobserver coefficients of variation for the measurement have been reported to be $8.4 \%$ and $10.0 \%$, respectively ${ }^{14)}$.

\section{Protocol of 64-Slice MDCT}

For assessments of calcium scoring, unenhanced cardiac CT was performed with a 64-detector row CT scanner (LightSpeed VCT; GE Medical System, Milwaukee, Wis) using prospective electrocardiography (ECG)triggering. The scanning range covered the entire heart from the level of the tracheal bifurcation to the diaphragm. The presence of calcification was determined according to the Agatston method ${ }^{15)}$ for multi-detector row CT with a $130-\mathrm{HU}$ threshold using a computer workstation (Card IQ, Advantage Workstation 4.3; GE Medical System). The vessel and CAC scores were independently determined by two radiologists and were averaged. The intra-assay coefficient of variation for the scoring was $<5 \%$.

Contrast-enhanced CT coronary angiography was performed with the same 64-detector row CT scanner using retrospective ECG-gating in a single breath hold. A 70-mL bolus of Iohexol (Omnipaque 350, $350 \mathrm{mg} \mathrm{I} / \mathrm{mL}$; GE Healthcare Ireland, Cork, Ireland) or Ioversol (Optiray 350, $350 \mathrm{mg} \mathrm{I} / \mathrm{mL}$; Tyco Healthcare, Montreal, Quebec, Canada) followed by $40 \mathrm{~mL}$ saline solution was continuously injected into an antecubital vein through an 18-gauge catheter at an injection rate of $5 \mathrm{~mL} / \mathrm{s}$. Transverse images were transferred to a computer workstation (Card IQ, Advantage Workstation 4.3; GE Medical System) for image post-processing. Two experienced observers ranked the image quality using a three-point ranking scale and analyzed all CT scans for signs of stenosis. In cases of disagreement between the two observers, a final decision was reached by consensus. Significant coronary stenosis was defined as a lumen diameter reduction of greater than $50 \%$ in any segment.

\section{Clinical and Laboratory Examinations}

The clinical examination included measurement of sitting blood pressure (with a random -zero sphygmomanometer), height, and weight. On the morning of the health examination, an overnight fasting blood sample was collected prior to the electron beam CT and brachial-ankle pulse wave velocity measurement. The blood samples were analyzed for the following parameters using standard techniques with commercial kits: total cholesterol, high-density lipoprotein cholesterol, low-density lipoprotein cholesterol, triglyceride, glucose, uric acid, and high-sensitivity 
Table 1. Basic characteristics of subjects with and without coronary artery disease

\begin{tabular}{|c|c|c|c|}
\hline Parameter & $\begin{array}{l}\text { Normal control } \\
\qquad(n=527)\end{array}$ & $\begin{array}{l}\text { Coronary artery disease } \\
\qquad(n=127)\end{array}$ & $p$-value \\
\hline Age (yrs) & $53.5 \pm 9.9$ & $58.8 \pm 9.1$ & $<0.001$ \\
\hline \multicolumn{4}{|l|}{ Gender } \\
\hline Female & $332(63.0)$ & $26(20.5)$ & \multirow{2}{*}{$<0.001$} \\
\hline Male & $195(37.0)$ & $101(79.5)$ & \\
\hline \multicolumn{4}{|l|}{ Waist circumference (m) } \\
\hline Female & $0.75 \pm 0.07$ & $0.84 \pm 0.07$ & $<0.05$ \\
\hline Male & $0.85 \pm 0.07$ & $0.92 \pm 0.08$ & $<0.05$ \\
\hline Body mass index $\left(\mathrm{kg} / \mathrm{m}^{2}\right)$ & $24.8 \pm 3.4$ & $26.1 \pm 3.8$ & $<0.05$ \\
\hline Mean blood pressure (mmHg) & $107.9 \pm 13.0$ & $115.8 \pm 16.1$ & $<0.001$ \\
\hline Cholesterol (mmol/L) & $5.27 \pm 0.98$ & $5.26 \pm 1.08$ & NS \\
\hline LDL-cholesterol (mmol/L) & $3.48 \pm 0.91$ & $3.66 \pm 0.97$ & 0.06 \\
\hline HDL-cholesterol (mmol/L) & $1.11 \pm 0.33$ & $1.00 \pm 0.23$ & $<0.001$ \\
\hline Triglyceride (mmol/L) & $1.42 \pm 1.00$ & $1.60 \pm 1.42$ & NS \\
\hline Glucose (mmol/L) & $5.57 \pm 1.34$ & $5.94 \pm 1.73$ & $<0.05$ \\
\hline hs-CRP (mg/L) & $0.21 \pm 0.36$ & $0.42 \pm 1.56$ & 0.02 \\
\hline ba-PWV (m/s) & $14.39 \pm 0.98$ & $15.94 \pm 3.07$ & $<0.001$ \\
\hline CAC score & $29.8 \pm 110.8$ & $293.1 \pm 435.9$ & $<0.001$ \\
\hline $\mathrm{ABI}$ & $1.21 \pm 0.29$ & $1.18 \pm 0.25$ & NS \\
\hline Hypertension $^{\mathrm{a}}$ & $220(41.7)$ & $94(74.0)$ & $<0.001$ \\
\hline Diabetes mellitus ${ }^{b}$ & $72(13.7)$ & $27(21.2)$ & $<0.001$ \\
\hline Hypercholesterolemia $^{c}$ & $204(38.7)$ & $50(39.4)$ & NS \\
\hline Smoking current & $105(19.9)$ & $34(26.8)$ & NS \\
\hline ex-smoker & $68(12.9)$ & $20(15.7)$ & \\
\hline
\end{tabular}

Continuous variables are expressed as the mean \pm SD. Categorical variables are expressed as $\mathrm{N}(\%)$. Abbreviations: LDL: low-density lipoprotein; HDL: high-density lipoprotein; hs-CRP: highly sensitive C-reactive protein; ba-PWV: brachialankle pulse wave velocity; CAC: coronary artery calcification; $\mathrm{ABI}$ : ankle-brachial pressure index

${ }^{a}$ Antihypertensive medication and/or high blood pressure ( $\geqq 140 \mathrm{mmHg}$ systolic or $\geqq 90 \mathrm{mmHg}$ diastolic); ${ }^{\mathrm{b}}$ Antidiabetic medication and/or fasting glucose $\geqq 7.0 \mathrm{mmol} / \mathrm{L}$; ${ }^{\mathrm{C}} \mathrm{Lipid}$-lowering medication and/or total cholesterol $\geqq 5.18 \mathrm{mmol} / \mathrm{L}$.

C-reactive protein (hs-CRP). The prediction of coronary heart disease was calculated using the Framingham risk score $(\mathrm{FRS})^{16)}$.

\section{Statistical Analysis}

All data are expressed as the mean \pm SD. Pearson correlation coefficients were used to assess the linear relationship between ba-PWV and the coronary artery calcium score. Differences in each variable between any two groups were evaluated using the $t$-test for continuous variables and the chi-square test for categorical variables. Because the distribution of the CAC scores was highly skewed, the common log-transformed CAC score $[\log (\mathrm{CAC}$ score +1$)]$ was used for further analysis. Comparisons of continuous data among three groups were based on analysis of variance (ANOVA). Multivariate logistic regression analysis was used to test the explanatory effect of each independent variable. The significant effect of each vari- able was determined by Wald statistics. Receiver operating characteristic (ROC) curves for coronary stenosis and ba-PWV, CAC score, and FRS were constructed and the area under the curve (AUC) was calculated. All of the analyses were conducted using the SAS software package for Windows, version 9.13. (SAS institute Inc., Cary, NC). $P$ values of $<0.05$ were considered significant.

We confirm that all applicable institutional and governmental regulations concerning the ethical use of human volunteers were followed during this research. Ethics approval for patient recruitment and analysis of data was obtained from the Institutional Review Board of the China Medical University Hospital in Taiwan.

\section{Results}

The 10-year risk of coronary artery disease calcu- 
Table 2. Age- and sex-adjusted odds ratios (OR) for coronary artery disease

\begin{tabular}{|c|c|c|c|}
\hline Parameter & $\begin{array}{l}\text { Normal Control } \\
\qquad(n=527) \\
\mathrm{N}(\%)\end{array}$ & $\begin{array}{l}\text { Coronary artery disease } \\
\qquad(n=127) \\
\mathrm{N}(\%)\end{array}$ & $\begin{array}{l}\text { adjusted OR } \\
(95 \% \mathrm{CI})\end{array}$ \\
\hline \multicolumn{4}{|l|}{ Body mass index } \\
\hline$<24\left(\mathrm{~kg} / \mathrm{m}^{2}\right)$ & $219(85.6)$ & $37(14.4)$ & Reference \\
\hline $24-27\left(\mathrm{~kg} / \mathrm{m}^{2}\right)$ & $231(81.6)$ & $52(18.4)$ & $1.33(0.84-2.11)$ \\
\hline$>27\left(\mathrm{~kg} / \mathrm{m}^{2}\right)$ & $77(68.2)$ & $36(31.8)$ & $2.77(1.63-4.69)$ \\
\hline \multicolumn{4}{|l|}{ Waist circumference } \\
\hline Normal & $320(83.6)$ & $63(16.4)$ & Reference \\
\hline Large $^{*}$ & $188(74.6)$ & $64(25.4)$ & $1.73(1.17-2.56)$ \\
\hline \multicolumn{4}{|l|}{ ba-PWV } \\
\hline$<14.0(\mathrm{~m} / \mathrm{s})$ & $225(86.9)$ & $34(13.1)$ & Reference \\
\hline $14.0-18.0(\mathrm{~m} / \mathrm{s})$ & $171(72.8)$ & $64(27.2)$ & $2.48(1.56-3.93)$ \\
\hline$>18.0(\mathrm{~m} / \mathrm{s})$ & $44(67.7)$ & $21(32.3)$ & $3.16(1.68-5.95)$ \\
\hline \multicolumn{4}{|l|}{ Hypertension } \\
\hline No & $307(90.3)$ & $33(9.7)$ & Reference \\
\hline Yes & $220(70.1)$ & $94(29.9)$ & $3.98(2.58-6.13)$ \\
\hline \multicolumn{4}{|l|}{ Diabetes Mellitus } \\
\hline No & $455(82.0)$ & $100(18.0)$ & Reference \\
\hline Yes & $72(72.7)$ & $27(27.3)$ & $1.71(1.04-2.80)$ \\
\hline
\end{tabular}

* Large waist circumference was defined as $\geqq 0.9 \mathrm{~m}$ (male) or $\geqq 0.8 \mathrm{~m}$ (female)

Abbreviations: CI: confidence interval; ba-PWV: brachial-ankle pulse wave velocity

lation using the Framingham risk score showed that $70 \%$ of the subjects were in the low-risk group, $20 \%$ in the moderate-risk group, and only $10 \%$ of the subjects were in the high-risk group. Of the 654 study subjects, $127(19.4 \%)$ were diagnosed as having at least one significant coronary stenotic lesion. Table $\mathbf{1}$ shows the basic characteristics of the normal controls and the subjects with coronary artery stenosis. The stenotic group had a higher mean age, body mass index, waist circumference, blood pressure, glucose level, and a higher proportion of men than the control group. There were no differences in total cholesterol, triglyceride levels or ankle-brachial pressure index (ABI) between the 2 groups; however, HDL-cholesterol was significantly lower in the stenotic group than in the control group. In addition, mean ba-PWV and the CAC score were significantly higher in the stenotic group than in the normal control group $(15.94 \pm 3.07$ $\mathrm{m} / \mathrm{s}$ vs. $14.39 \pm 0.98 \mathrm{~m} / \mathrm{s} ; 293.1 \pm 435.9$ vs. $29.8 \pm$ 110.8 , respectively; both $p<0.001$ ).

The age- and sex-adjusted OR for coronary artery stenosis was significantly higher in subjects with $\mathrm{BMI} \geqq 27 \mathrm{~kg} / \mathrm{m}^{2}$ (OR, 2.77; 95\% CI, 1.63-4.69) and central obesity (OR, 1.73; 95\% CI, 1.17-2.56) (Table 2). As expected, hypertension and diabetes mellitus were significantly associated with coronary stenosis (OR, 3.98; 95\% CI, 2.58-6.13 and OR, 1.71; 95\% CI,
1.04-2.80, respectively). The adjusted OR for coronary stenosis increased as ba-PWV increased ( $p$ for trend $=0.0001)$. Using ba-PWV $<14.0 \mathrm{~m} / \mathrm{s}$ as the reference group, we found that ba-PWV between $14.0-18.0 \mathrm{~m} / \mathrm{s}$ and ba-PWV $>18.0 \mathrm{~m} / \mathrm{s}$ were significantly associated with coronary stenosis (OR, 2.48; 95\% CI, 1.56-3.93 and OR, 3.16; 95\% CI, 1.68-5.95, respectively) (Table 3 ).

Pearson correlation analysis revealed that ba-PWV was significantly correlated with CAC score $(r=0.4, p<0.001)$, which increased as the ba-PWV increased. The mean CAC score was $35.7 \pm 173.6$ for ba-PWV $<14.0 \mathrm{~m} / \mathrm{s}, 100.2 \pm 249.7$ for ba-PWV between $14.0-18.0 \mathrm{~m} / \mathrm{s}$, and $227.6 \pm 412.5$ for ba-PWV $>18.0 \mathrm{~m} / \mathrm{s}(p<0.001$, ANOVA).

Using the ROC curve, we determined the optimal cutoff value of ba-PWV and CAC score that could predict the presence of coronary stenosis (Fig. 1). The cutoff value of ba-PWV at $15.64 \mathrm{~m} / \mathrm{s}$ had a sensitivity of $64.5 \%$, specificity of $65.6 \%$, and an AUC of 0.662 in predicting coronary artery stenosis. The cutoff value of the CAC score of 9.6 had a sensitivity of $80.0 \%$, specificity of $79.9 \%$, and an AUC of 0.853 in predicting coronary stenosis. We further analyzed the 10 -year risk of coronary heart disease calculated by the Framingham risk score (FRS). The cutoff point of FRS at $11.8 \%$ curve showed a sensitivity of $67.0 \%$, 


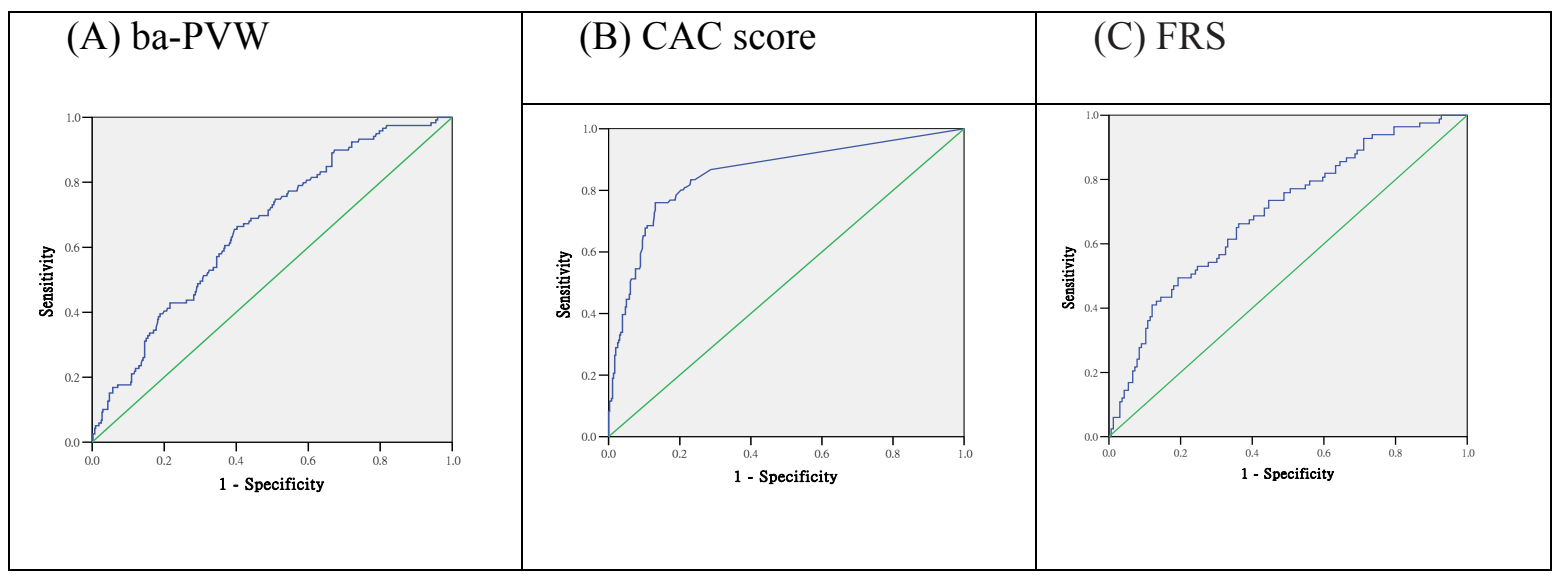

Fig. 1. Receiver operating characteristic (ROC) curve to determine the cutoff value and area under curve (AUC) for coronary artery stenosis by different indicators. (A). Cutoff point of brachial-ankle pulse wave velocity (baPWV) at $15.64 \mathrm{~m} / \mathrm{s}$ curve showed a sensitivity of $64.5 \%$, a specificity of $65.6 \%$, and an AUC of 0.662 . (B). Cutoff point of coronary calcification (CAC) score at 9.6 curve showed a sensitivity of $80.0 \%$, a specificity of 79.9\%, and an AUC of 0.853. (C). Cutoff point of Framingham risk score (FRS) at 11.8 curve showed a sensitivity of $67.0 \%$, a specificity of $71.1 \%$, and an AUC of 0.676 in predicting coronary stenosis.

Table 3. Trend for coronary calcification and significant coronary stenosis according to the severity of arterial stiffness measured as pulse wave velocity

\begin{tabular}{lcccc}
\hline & \multicolumn{3}{c}{ Brachial-ankle pulse wave velocity } & \multirow{2}{*}{$p$-value } \\
\cline { 2 - 4 } & $<14.0 \mathrm{~m} / \mathrm{s}$ & $14.0-18.0 \mathrm{~m} / \mathrm{s}$ & $>18.0 \mathrm{~m} / \mathrm{s}$ & \\
\hline Coronary artery Calcification score $^{\mathrm{a}}$ & $35.7 \pm 173.6$ & $100.2 \pm 249.7$ & $227.6 \pm 412.5$ & $<0.001$ \\
Coronary stenosis $^{\mathrm{N}}$ & & & & \\
$\quad$ No & $225(86.8)$ & $171(72.8)$ & $44(67.7)$ & $<0.001$ \\
Yes & $34(13.2)$ & $64(27.2)$ & $21(32.3)$ & \\
\hline
\end{tabular}

${ }^{a}$ Data expressed as the mean \pm SD and analyzed by analysis of variance (ANOVA) test

${ }^{\mathrm{b}}$ Data expressed as $\mathrm{N}(\%)$ and analyzed by Chi-square test.

specificity of $71.1 \%$, and an AUC of 0.676 . After combining FRS and ba-PWV, the AUC of the ROC curve increased to 0.728 . Ba-PWV had an additional power for correlating $\mathrm{CAD}$ to the Framingham risk score.

\section{Discussion}

Pulse wave velocity is an established index be arterial stiffness ${ }^{17)}$. In the present study, we demonstrated that ba-PWV correlated well with coronary atherosclerosis and stenosis in asymptomatic patients.

A few studies have addressed the relationship between ba-PWV and CAD in patients with suspected $\mathrm{CAD}$ or $\mathrm{CAD}$ equivalent. Tsuchiya $\mathrm{M}$ et al. found that stiffness in lower-leg arteries is associated with the severity of coronary calcification among asymptomatic type 2 diabetic patients. A cutoff point of ba-PWV at
$18.0 \mathrm{~m} / \mathrm{s}$ is a diagnostic value for identifying patients with the highest CAC score ${ }^{18)}$. Kim et al. revealed that ba-PWV at $16.35 \mathrm{~m} / \mathrm{s}$ had a sensitivity of $73 \%$ and specificity of $75 \%$ in detecting multiple coronary artery disease in Korean patients with diabetes ${ }^{19)}$. Imanishi $\mathrm{R}$ et al. revealed that a high ba-PWV level (mean: $18.9 \mathrm{~m} / \mathrm{s}$ vs. $15.2 \mathrm{~m} / \mathrm{s}$ ) is an independent predictor of the presence of CAD in men with suspected heart disease ${ }^{20)} . \mathrm{Xu}$ et al. showed that in suspected CAD patients, a ba-PWV $>18.0 \mathrm{~m} / \mathrm{s}$ often follows a severe coronary event ${ }^{21)}$. Our study revealed that in asymptomatic patients, ba-PWV at $15.64 \mathrm{~m} / \mathrm{s}$ had a sensitivity of $64.5 \%$, specificity of $65.6 \%$, and an AUC of 0.662 in predicting coronary artery stenosis. A similar result was found by Koji $\mathrm{Y}$ et al. in an asymptomatic Japanese population ${ }^{22)}$. These results indicate that the cutoff point of ba-PWV for detecting CAD may be lower in asymptomatic persons that in 
patients with suspected $\mathrm{CAD}$ or CAD equivalent.

The area under the ROC curve in this cross-sectional study indicates that the CAC score was better than ba-PWV in predicting the presence of CAD in asymptomatic patients. The values depicting the area under the ROC curve for ba-PWV were somewhat moderate in significance. A similar finding was reported by Tanaka $e t a l .{ }^{23)}$. The reason may be related to a lower cardiovascular risk in the Asian population. Further prospective longitudinal studies are warranted to properly address this issue.

Assessment of central arterial stiffness rather than peripheral arterial stiffness is more relevant to CAD risk stratification ${ }^{6,24)}$. Carotid-femoral PWV is the gold standard for assessing central arterial stiffness ${ }^{25)}$ : however, a high level of skill and exposure of the inguinal region are required for the measurement and its applicability is limited. The method we used is simple enough to use in clinical practice and community screening. A limitation of measuring the ba-PWV is that this parameter reflects not only elastic arterial stiffness but also muscular arterial stiffness ${ }^{14)}$. Nonetheless, ba-PWV and carotid-femoral PWV have both been shown to be associated with cardiovascular disease risk factors and clinical events ${ }^{23)}$. Recently, Tsuchikara $S$ et al. also reported that ba-PWV is an index of central arterial stiffness showing similar characteristics to those of central aortic PWV ${ }^{26)}$. Yu et al. reported that ba-PWV correlates better with LV mass and diastolic function and other indices of arterial function than carotid-femoral PWV, probably because ba-PWV encompasses a greater territory of the arterial tree than carotid-femoral PWV ${ }^{27}$.

Multislice computed tomography angiography is a non-invasive technique that can reliably detect coronary stenosis and quantify the degree of calcified plaque burden ${ }^{28)}$. Diffuse noncalcified plaques, which may result in blurred contours of the contrastenhanced lumen, and small noncalcified plaques were not included in this analysis; therefore, the presence of nonclacified plaques will be underestimated compared with histologic postmortem studies.

In our study, a similar distribution of demographic characteristics between participants and nonparticipants was found, indicating a random-type selection error. Thus, the biased results of the effect may be toward the null, indicating a lower threat to validity.

In conclusion, the present study revealed that arterial stiffness measured by ba-PWV correlated well with coronary atherosclerosis in a general population in Taiwan. Lifestyle modification, such as aerobic exercise and sodium restriction, is reported to be an effica- cious therapeutic intervention for preventing the progression of arterial stiffness ${ }^{29)}$. This non-invasive technique can assist in the early detection of cardiovascular disease and should be included in community screening programs.

\section{Conflict of Interest}

We confirm that all of our affiliations with or financial involvement in, within the past 5 years and foreseeable future, any organization or entity with a financial interest in or financial conflict with the subject matter or materials discussed in the manuscript have been completely disclosed.

\section{Funding}

This work was supported by China Medical University Hospital [CMU96-071] and the National Science Council [NSC93-2314-B-039-026].

\section{References}

1) Grundy SM, Pasternak R, Greenland P, Smith S Jr, Fuster $\mathrm{V}$ : Assessment of cardiovascular risk by use of multiplerisk-factor assessment equations: a statement for healthcare professionals from the American Heart Association and the American College of Cardiology. Circulation, 1999; 100: 1481-1492

2) Statistics on Cause of death in Taiwan. http://www.doh. gov.tw/CHT2006/DM/DM2_2.aspx?now_fod_list_no= $11663 \&$ class_no $=4408$ level_no $=5$ (access on March 23, 2010)

3) Cruickshank K, Riste L, Anderson SG, Wright JS, Dunn G, Gosling RG: Aortic pulse-wave velocity and its relationship to mortality in diabetes and glucose intolerance: an integrated index of vascular function? Circulation, 2002; 106: 2085-2090

4) Sutton-Tyrrell K, Najjar SS, Boudreau RM, Venkitachalam L, Kupelian V, Simonsick EM, Havlik R, Lakatta EG, Spurgeon H, Kritchevsky S, Pahor M, Bauer D, Newman A; Health ABC Study: Elevated aortic pulse wave velocity, a marker of arterial stiffness, predicts cardiovascular events in well-functioning older adults. Circulation, 2005; 111: 3384-3390

5) Lehmann ED: Clinical value of aortic pulse-wave velocity measurement. Lancet, 1999; 354: 528-529

6) Safar ME, Levy BI, Struijker-Boudier H: Current perspectives on arterial stiffness and pulse pressure in hypertension and cardiovascular diseases. Circulation, 2003; 107: 2864-2869

7) Zieman SJ, Melenovsky V, Kass DA: Mechanisms, pathophysiology, and therapy of arterial stiffness. Arterioscler Thromb Vasc Biol, 2005; 25: 932-943

8) Cockcroft JR, Wilkinson IB: Large arterial stiffness: an important therapeutic target. J Hum Hypertens, 2000; 14: $533-535$ 
9) Vlachopoulos C, Aznaouridis K, Stefanadis C: Prediction of cardiovascular events and all-cause mortality with arterial stiffness: a systematic review and meta-analysis. J Am Coll Cardiol, 2010; 55: 1318-1327

10) Rumberger JA, Sheedy PF, 3rd, Breen JF, Schwartz RS: Coronary calcium, as determined by electron beam computed tomography, and coronary disease on arteriogram. Effect of patient's sex on diagnosis. Circulation, 1995; 91: 1363-1367

11) Achenbach S, Ropers D, Hoffmann U, MacNeill B, Baum U, Pohle K, Brady TJ, Pomerantsev E, Ludwig J, Flachskampf FA, Wicky S, Jang IK, Daniel WG: Assessment of coronary remodeling in stenotic and nonstenotic coronary atherosclerotic lesions by multidetector spiral computed tomography. J Am Coll Cardiol, 2004; 43: 842-847

12) Tomiyama H, Koji Y, Yambe M, Shiina K, Motobe K, Yamada J, Shido N, Tanaka N, Chikamori T, Yamashina A: Brachial -- ankle pulse wave velocity is a simple and independent predictor of prognosis in patients with acute coronary syndrome. Circ J, 2005; 69: 815-822

13) Motobe K, Tomiyama H, Koji Y, Yambe M, Gulinisa Z, Arai T, Ichihashi T, Nagae T, Ishimaru S, Yamashina A: Cut-off value of the ankle-brachial pressure index at which the accuracy of brachial-ankle pulse wave velocity measurement is diminished. Circ J, 2005; 69: 55-60

14) Yamashina $A$, Tomiyama $H$, Takeda $K$, Tsuda $H$, Arai $T$, Hirose K, Koji Y, Hori S, Yamamoto Y: Validity, reproducibility, and clinical significance of noninvasive brachial-ankle pulse wave velocity measurement. Hypertens Res, 2002; 25: 359-364

15) Agatston AS, Janowitz WR, Hildner FJ, Zusmer NR, Viamonte M Jr, Detrano R: Quantification of coronary artery calcium using ultrafast computed tomography. J Am Coll Cardiol, 1990; 15: 827-832

16) Wilson PWF, D’Agostino RB, Levy D, Belanger AM, Silbershartz $\mathrm{H}$, Kannel WB: Prediction of coronary heart disease using risk factor categories. Circulation, 1998; 97: $1837-1847$

17) Tomiyama H, Yamashina A: Non-invasive vascular function tests: their pathophysiological background and clinical application. Circ J, 2010; 74: 24-33

18) Tsuchiya M, Suzuki E, Egawa K, Nishio Y, Maegawa H, Inoue S, Mitsunami K, Morikawa S, Inubushi T, Kashiwagi A: Stiffness and impaired blood flow in lower-leg arteries are associated with severity of coronary artery calcification among asymptomatic type 2 diabetic patients. Diabetes Care, 2004; 27: 2409-2415

19) Kim HJ, Nam JS, Park JS, Cho M, Kim CS, Ahn CW, Kwon HM, Hong BK, Yoon YW, Cha BS, Kim KR, Lee HC: Usefulness of brachial-ankle pulse wave velocity as a predictive marker of multiple coronary artery occlusive disease in Korean type 2 diabetes patients. Diabetes Res Clin Pract, 2009; 85: 30-34

20) Imanishi R, Seto $S$, Toda G, Yoshida M, Ohtsuru A, Koide Y, Baba T, Yano K: High brachial-ankle pulse wave velocity is an independent predictor of the presence of coronary artery disease in men. Hypertens Res, 2004; 27: 71-78

21) Xu Y, Wu Y, Li J, Ma W, Guo X, Luo Y, Hu D: The predictive value of brachial-ankle pulse wave velocity in coronary atherosclerosis and peripheral artery diseases in urban Chinese patients. Hypertens Res, 2008; 31: 10791085

22) Koji Y, Tomiyama H, Yamada J, Yambe M, Motobe K, Shiina K, Yamashina A: Relationship between arterial stiffness and the risk of coronary artery disease in subjects with and without metabolic syndrome. Hypertens Res, 2007; 30: 243-247

23) Tanaka H, Munakata M, Kawano Y, Ohishi M, Shoji T, Sugawara J, Tomiyama $\mathrm{H}$, Yamashina A, Yasuda $\mathrm{H}$, Sawayama T, Ozawa T: Comparison between carotidfemoral and brachial-ankle pulse wave velocity as measures of arterial stiffness. J Hypertens, 2009; 27: 20222027

24) Laurent S, Cockcroft J, Van Bortel L, Boutouyrie P, Giannattasio C, Hayoz D, Pannier B, Vlachopoulos C, Wilkinson I, Struijker-Boudier H; European Network for Non-invasive Investigation of Large Arteries: Expert consensus document on arterial stiffness: methodological issues and clinical applications. Eur Heart J, 2006; 27: 2588-2605

25) Adams MR, Robinson J, McCredie R, Seale JP, Sorensen KE, Deanfield JE, Celermajer DS: Smooth muscle dysfunction occurs independently of impaired endotheliumdependent dilation in adults at risk of atherosclerosis. J Am Coll Cardiol, 1998; 32: 123-127

26) Tsuchikura S, Shoji T, Kimoto E, Shinohara K, Hatsuda S, Koyama H, Emoto M, Nishizawa Y: Brachial- ankle pulse wave velocity as an index of central arterial stiffness. J Atheroscler Thromb, 2010; 17: 658-665

27) Yu WC, Chuang SY, Lin YP, Chen CH: Brachial-ankle vs carotid-femoral pulse wave velocity as a determinant of cardiovascular structure and function. J Hum Hypertens, 2008; 22: 24-31

28) Ohnesorge BM, Hofmann LK, Flohr TG, Schoepf UJ: CT for imaging coronary artery disease: defining the paradigm for its application. Int J Cardiovasc Imaging, 2005; 21: 85-104

29) Tanaka H, Safar ME: Influence of lifestyle modification on arterial stiffness and wave reflections. Am J Hypertens, 2005; 18: 137-144 